\title{
Estudo da Capacidade de Combinação de Linhagens de Matrizes de Frangos de Corte, por Meio da Análise de Fatores. Produção de Ovos ${ }^{1}$
}

\author{
Valéria Maria Nascimento Abreu ${ }^{2}$, Cosme Damião $\mathrm{Cruz}^{3}$, Martinho de Almeida e Silva ${ }^{4}$, Élsio \\ Antônio Pereira de Figueiredo ${ }^{5}$, Paulo Giovanni de Abreu ${ }^{6}$
}

\begin{abstract}
RESUMO - Com o objetivo de determinar a capacidade de combinação de linhagens de matrizes de corte, por meio da análise de fatores, cruzamentos (dialelo completo) dentro das linhagens fêmea (PP, KK e VV) foram usados. As características analisadas foram: número de ovos da $24^{\mathrm{a}}-32^{\mathrm{a}}(\mathrm{P} 1), 32^{\mathrm{a}}-40^{\mathrm{a}}(\mathrm{P} 2), 40^{\mathrm{a}}-48^{\mathrm{a}}(\mathrm{P} 3), 48^{\mathrm{a}}-56^{\mathrm{a}}(\mathrm{P} 4), 56^{\mathrm{a}}-64^{\mathrm{a}}(\mathrm{P} 5), 22^{\mathrm{a}}-66^{\mathrm{a}}(\mathrm{P} 6), 32^{\mathrm{a}}-66^{\mathrm{a}}(\mathrm{P} 7)$, $40^{\mathrm{a}}-66^{\mathrm{a}}(\mathrm{P} 8), 48^{\mathrm{a}}-66^{\mathrm{a}}(\mathrm{P} 9), 24^{\mathrm{a}}-40^{\mathrm{a}}(\mathrm{P} 10), 24^{\mathrm{a}}-48^{\mathrm{a}}(\mathrm{P} 11)$ e $24^{\mathrm{a}}-56^{\mathrm{a}}(\mathrm{P} 12)$ semana de idade. Na análise de fatores foram usados dois fatores, sendo os fatores de significado definidos após a rotação. O fator comum 1 apresentou o maior peso para a característica P6, sendo, então, considerado o fator de produção. Na seleção de progenitores superiores com base no valor genético-aditivo, o progenitor VV foi o que mais se destacou. O híbrido superior para as duas características mais importantes, produção no pico de postura e produção total de ovos, envolve os mesmos progenitores, o PP e VV. A análise de fatores forneceu fatores com significado biológico e econômico, possibilitando maiores facilidades de uso e interpretação.
\end{abstract}

Palavras-chave: análise de fatores, capacidade de combinação, matrizes de frango de corte

\section{Study of the Combining Ability of Broiler Matrix Lines by Means of Factor Analysis. Egg Production Traits}

\begin{abstract}
With the objective to determine the combining ability of broiler matrix lines by means of factor analysis, single crosses (full diallel) within female lines (PP, KK and VV) were used. The evaluated traits were egg numbers from the $24^{\text {th }}-32^{\text {nd }}(\mathrm{P} 1), 32^{\text {nd }}-40^{\text {th }}(\mathrm{P} 2)$, $\left.40^{\text {th }}-48^{\text {th }}(\mathrm{P} 3), 48^{\text {th }}-56^{\text {th }}(\mathrm{P} 4), 56^{\text {th }}-64^{\text {th }}(\mathrm{P} 5), 22^{\text {nd }}-66^{\text {th }}(\mathrm{P} 6), 32^{\text {nd }}-66^{\text {th }}(\mathrm{P} 7)\right), 40^{\text {th }}-66^{\text {th }}(\mathrm{P} 8), 48^{\text {th }}-66^{\text {th }}(\mathrm{P} 9), 24^{\text {th }}-40^{\text {th }}(\mathrm{P} 10), 24^{\text {th }}-48^{\text {th }}$ $(\mathrm{P} 11))$ and $24^{\text {th }}-56^{\text {th }}(\mathrm{P} 12)$ week of age. In the factor analysis, two factors were used, with the meaning biological factor defined after the factor rotation. The common factor 1 presented the bigger weight for the characteristic P6, being, then, considered the production factor. In the selection of superior progenitors based on the additive genetic value, the progenitor VV was the one that stood out. The superior hybrid for the two most important traits, peak of egg production and total egg production, involves the same progenitors, the PP and VV. The factor analysis provided factors with biological and economic meaning of easier use and interpretation.
\end{abstract}

Key Words: factor analysis, combining ability, broiler matrix lines

\section{Introdução}

A análise de fatores é uma técnica de análise multivariada que trata das relações internas de um conjunto de variáveis, substituindo um conjunto inicial de variáveis correlacionadas por um conjunto menor de fatores (ou variáveis hipotéticas), que podem ser nãocorrelacionados (fatores ortogonais) ou correlacionados (fatores oblíquos), que explicam a maior parte da variância do conjunto original (FACHEL, 1976).

Segundo POWELL e MACIVER (1977), objetivo geral da análise de fatores é reduzir uma série de variáveis a um pequeno número de fatores (outras variáveis) não-correlacionados e definidos somente pelas dimensões reais, sendo que os fatores resultantes retêm as informações mais importantes dos dados originais. Essa técnica permite estabelecer a existência ou não de algum padrão de relacionamento entre os dados, de tal modo que esses possam ser reordenados a um conjunto menor de fatores ou componentes (KIM, 1975). Suponha-se que as variáveis possam ser agrupadas por meio de suas correlações; procedendo-se assim, as variáveis de um mesmo grupo serão fortemente correlacionadas en-

\footnotetext{
${ }^{1}$ Parte do trabalho de tese de D.S. do primeiro autor, financiada pela CAPES/UFV/EMBRAPA.

2Pesquisador EMBRAPA/CNPSA, bolsista da CAPES.

${ }^{3}$ Professor da Universidade Federal de Viçosa.

${ }^{4}$ Professor da Universidade Estadual Norte Fluminense.

${ }^{5}$ Pesquisador EMBRAPA/CNPSA, bolsista do CNPq.

${ }^{6}$ PesquisadorEMBRAPA/CNPSA.
} 
tre si, mas pouco correlacionadas com variáveis de outro grupo. Cada grupo de variáveis será fator responsável pelas correlações observadas.

A análise de fatores e a análise por componentes principais podem ser vistas como técnicas que estudam a matriz de covariâncias das variáveis, sendo que o modelo da análise de fatores é mais elaborado (JOHNSON e WICHERN, 1982). Ambas iniciam-se com um modelo linear e encerram-se com a fatoração da matriz de covariâncias ou correlações. Contudo, na análise dos componentes principais, a fatoração da matriz é única, se todos os componentes de variância são diferentes. Entretanto, na análise de fatores, a fatoração da matriz não será única, pois, por transformação ortogonal (rotação), número infinito de matrizes de pesos fatoriais pode ser obtido para reproduzir a matriz de covariâncias ou correlações das variáveis respostas (QUEIROZ, 1984). Além disso, a equação básica do modelo linear dos componentes principais não possui componente residual, propriedade essa que a diferencia marcadamente da análise de fatores.

A análise de fatores tem sido muito usada em sistemas de classificação. KLOOSTERMAN e LAVHULICH (1972) apresentaram um método de interpretação estatística de dados de solo para uso agrícola e de engenharia, no qual a análise de fatores foi usada com base estatística para reduzir o número de variáveis a um número menor de fatores independentes, realizando, assim, a classificação dos solos, de acordo com seus valores financeiros, quanto aos seus possíveis usos.

FERNANDES e LIMA (1991) afirmam que a análise de fatores tem como objetivo básico a redução do número original de variáveis a um conjunto menor de "fatores" independentes, de tal forma que esses fatores possam explicar, de forma simples e reduzida, as variáveis originais. Na composição desses fatores, tem-se que:

- as variáveis mais correlacionadas combinam-se dentro do mesmo fator;

- as que compõem determinado fator são, praticamente, independentes das que constituem outro fator;

- a derivação dos fatores processa-se visando maximizar a porcentagem de variância total relativa a cada fator consecutivo; e

- os fatores não são correlacionados entre si.

Emprega-se a análise de fatores na fase inicial do trabalho de classificação, com o objetivo de identificar tipos a partir da ordenação e redução de variáveis, em dimensões básicas, que possibilitem o uso de técnicas que gerem e otimizem esquemas classificatórios, como análises de agrupamento e discriminante.

Este trabalho teve como objetivo determinar a capacidade de combinação de linhagens de matrizes de frango de corte, utilizando a análise de fatores, por meio de características de produção de ovos.

\section{Material e Métodos}

Os dados foram provenientes de experimento realizado no Setor de Melhoramento Genético de Aves (SMGA) do Centro Nacional de Pesquisa de Suínos e Aves (CNPSA), da Empresa Brasileira de Pesquisa Agropecuária (EMBRAPA), em Concórdia, Santa Catarina, no período de janeiro de 1994 a dezembro de 1995.

As aves deste experimento foram oriundas do cruzamento em dialelo completo dentro de três linhas maternas (KK, PP e VV), na Embrapa Suínos e Aves. O esquema de cruzamentos da linhas maternas foi o seguinte:

\begin{tabular}{cc}
\hline \multicolumn{2}{c}{ Progenitor } \\
\cline { 2 - 3 } Macho & Fêmea \\
Male & Female \\
\hline $1-\mathrm{PP}$ & $1-\mathrm{PP}$ \\
$2-\mathrm{KK}$ & $2-\mathrm{KK}$ \\
$3-\mathrm{VV}$ & $3-\mathrm{VV}$ \\
\hline
\end{tabular}

Destes cruzamentos em dialelo completo incluindo pais, híbridos e recíprocos, resultaram:

\begin{tabular}{cc}
\hline $\begin{array}{c}\text { Codificação } \\
\text { Code }\end{array}$ & $\begin{array}{c}\text { Cruzamento } \\
\text { Cross }\end{array}$ \\
\hline 11 & PP x PP \\
12 & PP x KK \\
13 & PP x VV \\
21 & KK x PP \\
22 & KK x KK \\
23 & KK x VV \\
31 & VV x PP \\
32 & VV x KK \\
33 & VV x VV \\
\hline
\end{tabular}

O delineamento experimental foi em blocos casualizados, consistindo nove cruzamentos, três blocos de 81 aves cada.

As 12 características de produção de ovos estudadas foram: número de ovos da $24^{\mathrm{a}}-32^{\mathrm{a}}$ (P1); $32^{\mathrm{a}}-40^{\mathrm{a}}(\mathrm{P} 2) ; 40^{\mathrm{a}}-48^{\mathrm{a}}(\mathrm{P} 3) ; 48^{\mathrm{a}}-56^{\mathrm{a}}(\mathrm{P} 4)$; $56^{\mathrm{a}}-64^{\mathrm{a}}(\mathrm{P} 5) ; 22^{\mathrm{a}}-66^{\mathrm{a}}(\mathrm{P} 6) ; 32^{\mathrm{a}}-66^{\mathrm{a}}(\mathrm{P} 7) ;$ $40^{\mathrm{a}}-66^{\mathrm{a}}(\mathrm{P} 8) ; 48^{\mathrm{a}}-66^{\mathrm{a}}(\mathrm{P} 9) ; 24^{\mathrm{a}}-40^{\mathrm{a}}(\mathrm{P} 10) ;$ $24^{\mathrm{a}}-48^{\mathrm{a}}(\mathrm{P} 11)$ e $24^{\mathrm{a}}-56^{\mathrm{a}}$ (P12) semana de vida das aves. Estas características foram obtidas por meio de anotações diárias do número de ovos.

Segundo CASTOLDI (1997), a análise de fatores procura explicar o comportamento de um número $\mathrm{p}$ 
260 Rev. bras. zootec.

de variáveis, em termos de número $\mathrm{m}(\mathrm{m}<\mathrm{p})$ de outras variáveis não-perceptíveis, chamadas fatores comuns. As análises foram realizadas utilizando-se o programa GENES (CRUZ, 1997), por meio do seguinte modelo:

$$
X_{j}=a_{j 1} F_{1}+a_{j 2} F_{2}+\ldots+a_{j m} F_{m}+\varepsilon_{j}
$$

em que

$X_{j}=$ variável $j$, para $j=1,2, \ldots, p$;

$\mathrm{a}_{\mathrm{ji}}=$ carga associada à variável $\mathrm{j}$ no fator $\mathrm{i}, \mathrm{i}=1,2, \ldots$ m;

$\mathrm{F}_{\mathrm{i}}=\mathrm{o}$ fator $\mathrm{i}$ comum a todas variáveis, os $\mathrm{m}$ fatores comuns não são correlacionados; e

$\mathrm{e}_{\mathrm{j}}=$ fator especifico da variável $\mathrm{j}$.

Na forma matricial:

$$
X_{p x 1}=\Lambda_{p x m} F_{m x 1}+\varepsilon_{p x 1}
$$

As pressuposições associadas ao modelo de fatores, dentro da configuração chamada de modelo de fatores ortogonais, considerando-se os fatores nãocorrelacionados, são:

(1) $\mathrm{E}(\mathrm{F})=0_{\mathrm{mx} 1}$;

(2) $\operatorname{COV}(\mathrm{F})=\mathrm{E}\left(\mathrm{FF}^{\prime}\right)=\mathrm{I}_{\mathrm{m} \times \mathrm{m} \text {; }}$

(3) $\mathrm{E}(\mathrm{e})=0_{\mathrm{p} \times 1}$;

(4) $\operatorname{COV}(\mathrm{e})=\mathrm{E}\left(\mathrm{ee}^{\mathrm{c}}\right)=\mathrm{Y}_{\mathrm{p} \text { x p }}$, em que $\mathrm{Y}$ é uma matriz diagonal; $\mathrm{e}$

(5) $\operatorname{COV}(\mathrm{e}, \mathrm{F})=\mathrm{E}(\mathrm{e}, \mathrm{F})=0_{\mathrm{p} x \mathrm{~m}}$.

Para melhor entendimento da análise de fatores, são necessárias as seguintes explicações:

- o objetivo da análise de fatores é representar cada fator como combinação linear de diversas variáveis subjacentes (FERNANDES e LIMA 1991);

- as variáveis mais explicadas, as que apresentam valores altos para as "cargas fatoriais", podem ser englobadas em um mesmo fator. Daí a redução do número inicial de variáveis para o número menor de fatores de explicação;

- a soma das "cargas fatoriais" ao quadrado, para cada variável, a "comunalidade", indica a proporção de variância total de cada variável, explicada pelo conjunto de fatores comuns. Então, define-se como comunalidade de uma variável $X_{j}$ a expressão algébrica dada por: $a_{j 1}^{2}+a_{j 2}^{2}+\ldots+a_{j m}^{2}$, que determina a fração da variância de $X_{j}$ dada pela influência dos fatores comuns, e como especificidade de $X_{j}$ a fração da variância de $X_{j}$ dada pela ação dos fatores específicos; e

- os "escores fatorais" são estimativas dos valores apresentados, para cada fator comum e observação.

A análise de fatores envolve vários estádios:

1. estabelecimento do número de fatores comuns a ser usado;

2. cálculo das cargas iniciais desses fatores;

3. rotação das cargas iniciais, obtendo-se, com isso, as cargas finais, que permitem definir o fator desejado (fator de produção); e

4. estimação dos escores fatoriais associados a cada variável.

\section{Determinação do número de fatores comuns}

O método utilizado para determinar o número de fatores foi escolher tantos fatores comuns quantos forem os autovalores maiores ou iguais à unidade, obtidos da matriz de correlação genética das variáveis originais (Harman, 1968, citado por CASTOLDI, 1997).

\section{Cálculo das cargas fatoriais}

As cargas fatoriais de cada variável são dadas por:

em que

$$
a_{i j}=\sqrt{\lambda_{i}} V_{i j}
$$

$\mathrm{a}_{\mathrm{ij}}=$ carga fatorial do fator $\mathrm{i}$ correspondente à variável $\mathrm{j}$;

$\sqrt{\lambda_{\mathrm{i}}}=$ autovalor i maior que a unidade, obtido da matriz de correlações genéticas; e

$\mathrm{V}_{\mathrm{ij}}=$ valor $\mathrm{j}$ do autovetor $\mathrm{i}$.

Em síntese, cada autovalor maior que a unidade corresponde a um autovetor, que, por sua vez, é constituído por um número de valores iguais ao número de variáveis originais. Logo, um mesmo fator terá cargas individuais para todas as variáveis, o que esclarece a razão da denominação "fator comum", empregada na teoria de "factor analysis".

\section{Rotação fatorial}

O principal objetivo da rotação de fatores é obter uma matriz, mais facilmente interpretável ou mais identificável com a natureza das variáveis. São feitas rotações o suficiente para se obter uma estrutura mais simples, em que cada variável, tanto quanto possível, se correlacione significativamente com um fator. Não importando quantos fatores comuns sejam utilizados, a rotação é feita sempre de dois a dois, em um total de $\mathrm{C}_{\mathrm{m}}^{2}$ rotações em que $\mathrm{m}$ é o número de fatores comuns (CASTOLDI, 1997).

\section{Cálculo dos escores fatoriais}

$\mathrm{O}$ escore fatorial quantifica o efeito de cada fator comum na expressão dos caracteres, que foram obtidos de acordo com Manly (1980), citado por CASTOLDI (1997), da seguinte forma:

$$
F^{*}=\left(G G^{\prime}\right)^{-1} G^{\prime} X
$$

em que

$\mathrm{F}^{*}=$ vetor de dimensão $\mathrm{m} \times 1$ de escores fatoriais; 
$\mathrm{G}=$ matriz de dimensão $\mathrm{p} \times \mathrm{m}$ das cargas fatoriais rotacionadas; e

$\mathrm{X}=$ vetor de dimensão $\mathrm{p} \times 1$ de médias dos caracteres.

Em seguida, foi realizada a análise (univariada) dialélica dos escores fatoriais, sendo determinados as estimativas da capacidade geral de combinação (CGC - $\hat{\mathrm{g}}_{\mathrm{i}}$ ) e da capacidade específica de combinação $\left(\mathrm{CEC}-\hat{\mathrm{s}}_{\mathrm{ii}}\right.$ e $\left.\hat{\mathrm{s}}_{\mathrm{ij}}\right)$, o efeito recíproco $\left(\operatorname{Rec}-\hat{\mathrm{r}}_{\mathrm{ij}}\right)$ e a média dos cruzamentos (F1 e recíproco).

\section{Resultados e Discussão}

Os autovalores e as porcentagens de explicação simples e acumulada dos fatores são apresentados na Tabela 1. Fizeram parte da análise da capacidade combinatória somente os fatores com autovalores maiores que a unidade (FAT1 e FAT2).

Estão apresentados, na Tabela 2, os valores das cargas iniciais (CFI), as cargas após a rotação (CFR) e as comunalidades (COM) das características.

Um mesmo fator terá cargas individuais para todas as características, daí a denominação de fator comum. Um dos principais objetivos da análise de fatores é a caracterização dos fatores comuns, a qual é função dos caracteres com maior carga dentro desses fatores. Um fator só poderá ser caracterizado com clareza se existirem alguns caracteres com altas cargas fatoriais e que ainda tenham alguma relação de funcionalidade entre si. As características mais explicadas, as que apresentam valores altos para as cargas fatoriais, podem ser englobadas em um mes-

Tabela 1 - Autovalores, percentagem total e acumulada da variância dos fatores

Table 1 - Eigenvalues, percentage of total and accumulated variance of the factors

\begin{tabular}{lccc}
\hline $\begin{array}{l}\text { Fator } \\
\text { Factor }\end{array}$ & $\begin{array}{c}\text { Autovalor } \\
\text { Eigenvalue }\end{array}$ & $\begin{array}{c}\text { Porcentagem } \\
\text { total }(\%) \\
\text { Percentage } \\
\text { of the total }\end{array}$ & $\begin{array}{c}\text { Porcentagem } \\
\text { acumulada }(\%) \\
\text { Accumulated } \\
\text { percentage }\end{array}$ \\
\hline FAT1 & 7,985514 & 66,54 & 66,54 \\
FAT2 & 3,830199 & 31,92 & 98,46 \\
FAT3 & 0,138591 & 1,15 & 99,61 \\
FAT4 & 0,040215 & 0,33 & 99,95 \\
FAT5 & 0,005259 & 0,04 & 99,99 \\
FAT6 & 0,000143 & 0,01 & 100,00 \\
FAT7 & 0,000110 & 0,00 & 100,00 \\
FAT8 & 0,000077 & 0,00 & 100,00 \\
FAT9 & 0,000058 & 0,00 & 100,00 \\
FAT10 & 0,000002 & 0,00 & 100,00 \\
FAT11 & 0,000069 & 0,00 & 100,00 \\
FAT12 & 0,000106 & 0,00 & 100,00 \\
\hline
\end{tabular}

mo fator de explicação. Algumas vezes, porém, ao se assumirem valores semelhantes, as cargas dos fatores não se apresentam distintas entre si, impedindo, com isso, perfeita caracterização dos fatores. A maneira de superar essa dificuldade é por intermédio da rotação dos fatores. A partir das cargas fatoriais iniciais, obtêm-se as cargas finais, de forma que algumas características terão cargas fatoriais próximas de 1,0 (um) e as restantes próximas de zero, permitindo definir o fator de produção (fator biológico), tornando mais clara a interpretação dos fatores.

Dessa forma, todas as características, exceto P1 e P10, podem ser englobadas no fator 1 e as características P1, P2, P6, P10, P11 e P12, no fator 2. Após rotação das cargas iniciais, para obtenção das cargas finais, pode-se observar que o fator comum que apresentou maior peso para a característica P6, que é considerada de maior interesse neste estudo, foi o fator 1, que é, então, considerado o fator de produção sobre o qual a seleção será realizada. Então, o fator 1 é o determinante da produção total de ovos e o fator 2, determinante do pico de postura.

As comunalidades indicam a proporção de variância total de cada variável explicada pelo conjunto de fatores comuns (Tabela 2). Os escores fatoriais (Tabela 3) quantificam o efeito de cada fator comum na expressão dos caracteres.

Os maiores escores, para o primeiro fator, foram obtidos pelos cruzamentos 13 e 33. Já para o segundo fator, os maiores escores foram os dos cruzamentos $11 \mathrm{e}$ 13. Dessa forma, a melhor maneira de se selecionarem os cruzamentos é por meio do estudo da CGC das linhagens.

As estimativas da CGC $\left(\hat{\mathrm{g}}_{\mathrm{i}}\right), \operatorname{CEC}\left(\hat{\mathrm{s}}_{\mathrm{ii}}\right.$ e $\left.\hat{\mathrm{s}}_{\mathrm{ij}}\right) \mathrm{e}$ efeito recíproco $\left(\hat{\mathrm{r}}_{\mathrm{ij}}\right)$ e a média dos cruzamentos $(\mathrm{F} 1$ e recíproco), obtidas a partir da análise dos escores dos dois fatores, estão apresentadas na Tabela 4.

Pelo Fator 1 (FAT1) seria selecionado pela CGG o progenitor 3. Já pelo Fator 2 (FAT2), os progenitores 1e 3 seriam selecionados. A seleção de híbridos superiores pelos dois fatores mostra que híbrido 13 seria selecionado para se obter melhoria nas características representas nestes fatores.

A técnica de análise de fatores não tem sido usada para este fim, entretanto, por intermédio deste trabalho, pode-se inferir que esta pode ter potencial em estudos genéticos, pois engloba várias características e permite a obtenção de fatores com significados biológicos e econômicos. Portanto, ao se selecionarem linhas, dentro do fator de produção, para melhorar certa característica, o melhorista tem o conhecimento de como as outras características 
262 Rev. bras. zootec.

Tabela 2 - Valores das cargas fatoriais ${ }^{a}$ iniciais (CFI), após rotação (CFR) e comunalidades (COM) das características

Table 2 - Values for the initial factors charge (CFI), after rotation (CFR) and comunility (COM) of the characteristics

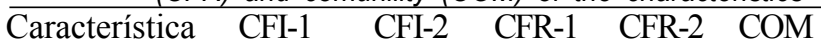

Characteristic

\begin{tabular}{llllll}
\hline P1 & 0,2102 & 0,9726 & $-0,2776$ & $\mathbf{0 , 9 5 5 7}$ & 0,9903 \\
P2 & 0,9122 & 0,3500 & 0,6361 & 0,7418 & 0,9548 \\
P3 & 0,9150 & $-0,3339$ & 0,9638 & 0,1413 & 0,9488 \\
P4 & 0,8965 & $-0,4110$ & 0,9842 & 0,0648 & 0,9727 \\
P5 & 0,8856 & $-0,4321$ & 0,9846 & 0,0410 & 0,9711 \\
P6 & 0,9599 & 0,2727 & $\mathbf{0 , 7 1 4 8}$ & 0,6964 & 0,9959 \\
P7 & 0,9555 & $-0,2931$ & 0,9800 & 0,1966 & 0,9990 \\
P8 & 0,9057 & $-0,4219$ & 0,9975 & 0,0595 & 0,9985 \\
P9 & 0,8906 & $-0,4417$ & 0,9936 & 0,0350 & 0,9884 \\
P10 & 0,4111 & 0,9107 & $-0,0713$ & 0,9967 & 0,9984 \\
P11 & 0,6226 & 0,7822 & 0,1757 & 0,9842 & 0,9996 \\
P12 & 0,8233 & 0,5651 & 0,4556 & 0,8887 & 0,9973
\end{tabular}

a $\mathrm{O}$ número de cargas fatoriais corresponde ao número de autovalores maiores que a unidade, obtidos a partir da matriz de correlações entre as variáveis originais.

a The number of factor changes corresponds to the number of eigenvalues greater than unit obtained from the matrix of correlation of the observed traits. 1 - FAT1.

2- FAT2.

1 - Factor 1.

2 - Factor 2.
Tabela 3 - Escores para os dois fatores, de acordo com os cruzamentos

Table 3 - Scores for the two factors, according to the crosses

\begin{tabular}{llc}
\hline \multirow{2}{*}{$\begin{array}{l}\text { Cruzamento } \\
\end{array}$} & \multicolumn{3}{c}{$\begin{array}{c}\text { Escore } \\
\text { Score }\end{array}$} \\
\cline { 2 - 3 } & FAT1 & FAT2 \\
11 & 584,0427 & $\mathbf{4 9 6 , 6 9 6 9}$ \\
12 & 608,4260 & 479,5202 \\
13 & $\mathbf{6 2 6 , 4 0 1 8}$ & $\mathbf{5 0 3 , 2 0 5 4}$ \\
21 & 557,9036 & 449,4997 \\
22 & 574,0950 & 445,2876 \\
23 & 583,5186 & 461,1284 \\
31 & 604,4451 & 497,0684 \\
32 & 616,5853 & 462,8853 \\
33 & $\mathbf{6 3 7 , 6 5 3 7}$ & 481,1057 \\
\hline
\end{tabular}

Tabela 4 - Estimativas da capacidade geral de combinação $\left(\hat{\mathrm{g}}_{\mathrm{i}}\right)$, capacidade especifica de combinação ( $\hat{\mathrm{S}}_{\mathrm{ii}}$ e $\left.\hat{\mathrm{S}}_{\mathrm{ij}}\right)$, efeito recíproco ( $\left.\hat{\mathrm{r}}_{\mathrm{ij}}\right)$ e média ( $\mathrm{F} 1 \mathrm{e} \mathrm{Rec}$ ) obtidas a partir dos escores dos dois fatores

Table 4 - Estimates of general and specific combining ability, reciprocal effect and mean crosses ( $F 1$ and Rec) obtained from the scores of the two factors

\begin{tabular}{|c|c|c|c|c|c|c|c|c|}
\hline \multirow{3}{*}{$\begin{array}{l}\text { Cruzamento } \\
\text { Cross }\end{array}$} & \multicolumn{4}{|c|}{ FAT1 } & \multicolumn{4}{|c|}{ FAT2 } \\
\hline & \multicolumn{2}{|c|}{$\begin{array}{l}\text { Média } \\
\text { Mean }\end{array}$} & \multicolumn{2}{|c|}{$\begin{array}{l}\text { Efeito } \\
\text { Effect }\end{array}$} & \multicolumn{2}{|c|}{$\begin{array}{l}\text { Média } \\
\text { Mean }\end{array}$} & \multicolumn{2}{|c|}{$\begin{array}{l}\text { Efeito } \\
\text { Effect }\end{array}$} \\
\hline & F1 & Rec & $\hat{s}_{i j}$ & $\hat{\mathbf{r}}_{i j}$ & F1 & Rec & $\hat{s}_{i j}$ & $\hat{r}_{i j}$ \\
\hline$\overline{12}$ & 608,425 & 557,903 & 2,414 & 25,261 & 479,517 & 449,497 & $-4,717$ & 15,010 \\
\hline 13 & 626,401 & 604,444 & 2,733 & 10,978 & 503,202 & 497,065 & 7,094 & 3,068 \\
\hline \multirow[t]{2}{*}{23} & 583,518 & 616,585 & $-4,198$ & $-16,533$ & 461,125 & 462,882 & $-1,189$ & $-0,878$ \\
\hline & \multicolumn{2}{|c|}{$\begin{array}{l}\text { Média } \\
\text { Mean }\end{array}$} & $\hat{\mathrm{g}}_{\mathrm{i}}$ & $\hat{\mathrm{S}}_{\mathrm{ii}}$ & \multicolumn{2}{|c|}{$\begin{array}{c}\text { Média } \\
\text { Mean }\end{array}$} & $\hat{\mathrm{g}}_{\mathrm{i}}$ & $\hat{\mathrm{s}}_{\mathrm{ii}}$ \\
\hline 11 & \multicolumn{2}{|c|}{584,042} & $-5,020$ & $-5,148$ & \multicolumn{2}{|c|}{496,693} & 11,959 & $-2,377$ \\
\hline 22 & \multirow{2}{*}{\multicolumn{2}{|c|}{574,094}} & $-13,460$ & 1,784 & \multicolumn{2}{|c|}{445,284} & $-17,887$ & 5,906 \\
\hline 33 & & 637,653 & 18,480 & 1,464 & \multicolumn{2}{|c|}{481,102} & 5,928 & $-5,905$ \\
\hline
\end{tabular}

se englobam dentro deste fator e quais também serão melhoradas simultaneamente.

\section{Conclusões}

$\mathrm{O}$ fator comum que apresentou maior peso para a característica P6 foi o fator 1, que foi considerado o fator de produção ou fator determinante da produção total de ovos. Já o fator 2 foi determinante do pico de postura.

Os progenitores 1 e 3 foram selecionados pela CGC para os dois fatores.

A análise de fatores parece ter grande potencial em estudos genéticos, pois, além de estudar conjun- tamente várias características, fornece fatores com significados biológicos e econômicos, possibilitando maiores facilidades de uso e interpretação.

\section{Referências Bibliográficas}

CASTOLDI, F. L. Estudo comparativo de diversos métodos de seleção multivariada em milho (Zea mays L.). Viçosa, MG, UFV. 1997. Tese (Doutorado em Melhoramento Genético) Universidade Federal de Viçosa, 1997.

CRUZ, C. D. 1997. Programa GENES-Aplicativo computacional em genética e estatística. Viçosa, MG: UFV, 442p.

FERNANDES, T. A. G., LIMA, J. E. 1991. Uso de análise multivariada para identificação de sistemas de produção. Pesq. Agrop. Bras., 26(10):1823-1836. 
JOHNSON, R. A., WICHERN, D. W. 1982. Applied multivariate statistical analysis. 1. ed. Englewood Cliffs, Prentice Hall, 594p.

KIM, J. O. 1975. Factor anlysis. In: NIE, H.H, HULL, C. H., JENKINS, J. G., STEIBRENNER, K., BENT, D.H (eds), statistical package for the social sciences. 2 ed., New York, McGraw-Hill. Inc... p 468-514.

POGGENPOEL, D. G., DUCKITT, J. S. 1988. Genetic basis of the increase in egg weight pullet age in white leghorne flock. Br. Poult. Sci., 29(4):863-867.

QUEIROZ, W. T. Análise de fatores ("Factor analysis") pelo método da máxima verossimilhança: aplicação do estudo da estrutura de florestas tropicais. Piracicaba, SP, ESALQ, 1984. 112p. Tese (Doutorado em Melhoramento Genético) - Escola Superior de Agricultura "Luis de Queiroz", 1984.

Recebido em: 25/06/98

Aceito em: 15/09/98 